\title{
In-flight Identification of the Augmented Flight Dynamics of the Rmax Unmanned Helicopter
}

Simone Duranti and Gianpaolo Conte

Linköping University Post Print

N.B.: When citing this work, cite the original article.

Original Publication:

Simone Duranti and Gianpaolo Conte, In-flight Identification of the Augmented Flight Dynamics of the Rmax Unmanned Helicopter, 2007, 17th IFAC Symposium on Automatic Control in Aerospace.

http://dx.doi.org/10.3182/20070625-5-FR-2916.00038

Copyright: International Federation of Automatic Control

Postprint available at: Linköping University Electronic Press

http://urn.kb.se/resolve?urn=urn:nbn:se:liu:diva-75781 


\title{
IN-FLIGHT IDENTIFICATION OF THE AUGMENTED FLIGHT DYNAMICS OF THE RMAX UNMANNED HELICOPTER
}

\author{
Simone Duranti and Gianpaolo Conte
}

\author{
Autonomous UAV Technology Laboratory (UAVTech) \\ Artif. Intelligence and Int. Comp. Systems Div. (AIICS) \\ Department of Computer and Information Science \\ Linköping University, SE-58183 Linköping, Sweden
}

\begin{abstract}
The flight dynamics of the Yamaha RMAX unmanned helicopter has been investigated, and mapped into a six degrees of freedom mathematical model. The model has been obtained by a combined black-box system identification technique and a classic model-based parameter identification approach. In particular, the closed-loop behaviour of the built-in attitude control system has been studied, to support the decision whether to keep it as inner stabilization loop or to develop an own stability augmentation system. The flight test method and the test instrumentation are described in detail; some samples of the flight test data are compared to the model outputs as validation, and an overall assessment of the built-in stabilization system is supplied.
\end{abstract}

Keywords: Helicopter Dynamics, Closed-Loop Identification.

\section{BACKGROUND}

The UAVTech research group at Linköping University has chosen the RMAX industrial robot as flying platform for experimenting on intelligent autonomous vehicles and developing information technology for Unmanned Aerial Vehicles (UAVs). The particular operational environment that the research focuses on consists of roads carrying automobile traffic and urban environments in general, which put tight requirements on the manoeuvrability of the aircraft. The urban environment set a strict requirement on the navigation precision, since flight close to buildings and obstacles in general at low altitude is required. Moreover, the typical manoeuvring task consists of tracking a car, while avoiding the aforementioned obstacles. Therefore demanding requirements are set also on the manoeuvrability of the vehicle, that has to operate in a wide speed envelope and within corridors that are not many times wider then the rotor diameter. To aid the control system development and support the related flight-testing, a flight dynamics simulator has been developed, derived directly from experimental data with a hybrid technique embodying both black-box identification concepts and classic parameter identification methods.

\section{THE PLATFORM}

The RMAX industrial robot (Fig. 1, ref. (5 )) is a conventional tail rotor configuration helicopter, $3.6 \mathrm{~m}$ long and with a $3.1 \mathrm{~m}$ diameter main rotor, weighing $61 \mathrm{~kg}$ empty, and up to $95 \mathrm{~kg}$ when fully loaded . It is widely used in Japan for crop spraying and is presently the most produced and successful UAV on the civil market. Many reasons contributed to this success, and one of them is surely its easiness of operation, that enables inexperienced users to operate it after a short piloting 


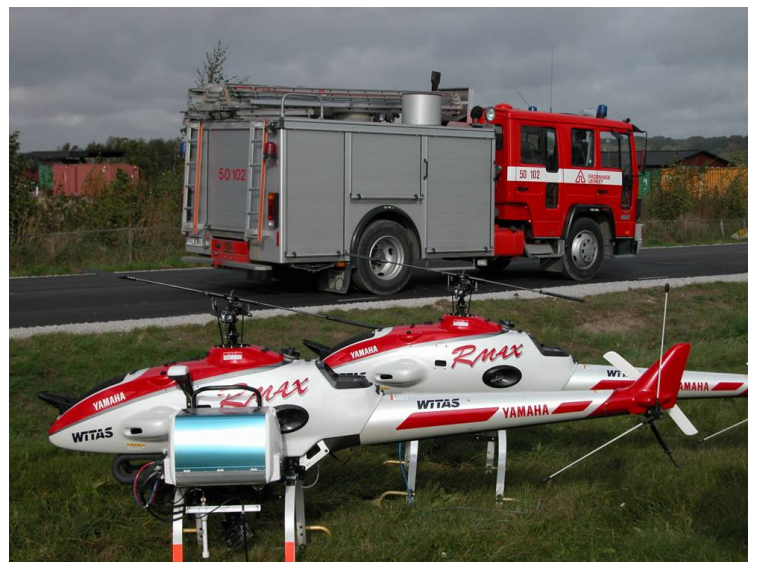

Fig. 1. The two RMAX helicopters operated by the UAVTech research group at Linköping University

course. This is a consequence of the digital stabilization system developed by Yamaha Motor Co., consisting of an attitude control system (YACS) that also augments the vertical dynamics, providing stability and reducing sensitivity to gusts. The YACS can be engaged/disengaged during flight with a push button on the radio transmitter: when disengaged, the pilot has direct authority on the servos, as a normal model helicopter, while when the YACS is engaged the digital controller augments the action of the pilot. Several control strategies have been under investigation within the WITAS control group; the well-proven built in attitude control system to be used as internal loop is an appealing solution, since it provides the stability that the nude platform misses and allows to rise the control problem directly to the navigation level and to address the autonomy issues. On the other hand, the exact behaviour of the YACS was initially unknown, since the control laws and the source code are obviously handled as company confidential information by Yamaha. In the Flight Manual a very limited flight envelope is recommended, mainly consisting of hovering and slow translation flight, which are the only modes of practical use for crop spraying applications. Detail information about the behaviour of the YACS outside this restricted envelope was missing. This made it difficult to assess if the YACS could be a proper choice as inner stabilizing loop, and at the same time the interface with the upper navigation layers was somehow undefined, since the control inputs to the YACS were unknown.

The identification of a simulation model of the augmented flight dynamics of the RMAX YACSIn-the-Loop (YIL) has therefore been considered the most straightforward solution to:

- map the performance and the robustness of the YACS on the envelope of interest, to support the decision whether to adopt it as inner loop or to develop an own stabilization system interfacing directly to the servos;

- define the interface to the upper layer of the control system, in case that YACS was adopted;

- build upper control layers in a more efficient way, avoiding as much as possible on-the-field empirical tuning of the control gains.

\section{IDENTIFICATION STRATEGY}

Two dynamic systems compose the closed-loop system under investigation (Fig.2):

- The un-augmented helicopter flight dynamics: the inputs are the commands sent to the servos and the output the state of the helicopter; the servo, rotor/flybar and rigid body dynamics are parts of this block;

- The YACS: it receives as external inputs the pilot controls and as internal inputs the sensor information about the state of the helicopter (load factors, angular rates and attitude angles), and sends commands to the servos.

The first block could have been identified with high precision with a classic parameter identification approach. The model structure of a sub-scale helicopter doesn't differ substantially from fullscale helicopters, and even the Bell-Hiller mechanism dynamics (very common on sub-scale helicopters) is described in detail on literature (Ref. (4)). Despite the scientific interest in such an exercise, this wouldn't have solved the problem, since the YACS dynamics would have still been unknown, such as the resulting closed loop behaviour, and no elements were available to make reasonable hypotheses on the structure of the model

Therefore a hybrid black-box identification approach has been adopted, under the following assumptions:

- The input to the system is a set a four variables, corresponding to the four controls available to the pilot on the radio-control,

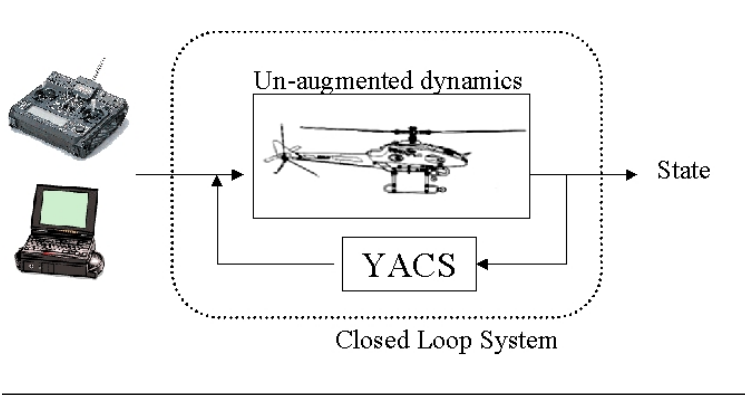

Fig. 2. Closed loop system 
namely longitudinal (ELE), lateral (AIL), pedal (RUD) and collective (THR);

- The four input variables control respectively the pitch angle, the roll angle, the vertical velocity and the yaw rate;

- The four channels, when considering the YIL behaviour, are uncoupled by the YACS;

- The non-linearity of the YACS can be wrapped into a non-linear gain shaping the input command;

- The rotor flapping dynamics is filtered out by the YACS and can be neglected; the rotor disk is assumed to be rigidly connected to the helicopter body (rigid-body approximation).

The three first assumptions have been based on the first impressions reported by the pilot after the first test flights. The identification strategy is "hybrid" in the sense that a black-box approach has been adopted to identify the dynamics involving the YACS, while parameter identification has been used to determine those few remaining parameters describing the flight dynamics once the attitude angles were given.

\section{TEST INSTRUMENTATION}

The core of the test instrumentation is a 266 $\mathrm{MHz} \mathrm{PC} 104$ Pentium P5, that in the current architecture performs even the strict real-time control tasks. The I/O consists of 11 RS232 serial channels (one modified for non-standard baud rate) and of an auto calibrating analogue board (16 differential analogue input channels, $24 \mathrm{I} / \mathrm{O}$ digital inputs). The data are logged in the 24 MB volatile RAM (no hard-disk is used), and the logging function can be triggered from ground to optimise the recording time, that is of about 30 min. The sensors' information is provided by a Systron Donnier Digital Quartz INS/GPS, providing raw information about load factors and angular rates $(100 \mathrm{~Hz}$ ), and a GPS (with differential correction from a Leica reference station) corrected navigation solution consisting of Euler angles $(10 \mathrm{~Hz})$, horizontal/vertical ground speed and position/altitude $(10 \mathrm{~Hz})$, by a static pressure gauge $(40 \mathrm{~Hz})$, and a Honeywell HMR3000 digital compass $(15 \mathrm{~Hz})$; the YAS sensors (load factors and Euler angles at $67 \mathrm{~Hz}$ and angular rates at $200 \mathrm{~Hz}$ ) are also available, such as a Mekatronix MUST A/40 sonar $(10 \mathrm{~Hz})$ for precise low altitude measurement during takeoff and landing, despite not used for the identification process. Most of the test activity herein described has been conducted in the early stages of the development process, when the INS/GPS was not available yet. Therefore the analysis herein reported is based on the information gained by the YAS sensors (load factors, angular rates and calculated Euler angles) and by a pressure altimeter.

\section{FLIGHT TESTS}

Flight tests have been initially conduced with the pilot manually controlling the helicopter, sending frequency sweeps, steps and doublets through the sticks. After the evaluation of the first results, Flight Test Functions (FTF) have been introduced instead, that are pre-programmed stick input sequences sent to the system by the onboard Central Computer (CC) and activated by the pilot through a push button on the radio control. With this technique, the role of the pilot has been limited in stabilising the helicopter on the operating point, activating the FTF and taking over to manual control at the end of the test window or when predefined abort criteria were met. The input amplitude has been so far limited to $40 \%$ of the maximum available in the longitudinal, lateral and yaw channel, and to $+40 \% /-20 \%$ in the vertical channel. So far, the hovering condition has been used as operating point for frequency sweeps and steps, that have been hold up to the maximum distance that allowed a safe manual recover, to identify the steady state response; this resulted in time windows never exceeding $15 \mathrm{~s}$, that indicated the need of a different approach for tests requiring stabilised flight at higher speed. In fact, cruise-flight experiments are now being performed with a different test method: a car is used as chase vehicle, to enable the pilot to be always within a reasonable distance from the helicopter, and FTF have been integrated with closed-loop control, to achieve stabilised operating points and altitude holding. Besides the problem of keeping the helicopter in sight, it has emerged that keeping constant altitude and speed by manual control is an unrealistic task. Despite the dynamics of the system has been identified only in the hovering region, the results seem to fit very well even to the higher speed range: the reason of that can be identified in the linearising effect of the YACS, that filters out the variations of the stability derivatives on the speed envelope.

\section{DATA EVALUATION}

Routines for the conversion of the data files into a Matlab compatible format have been created. The data are downloaded after landing through a TCP/IP connection, in binary format, converted to ASCII files (one for each sensor, each with the own time vector), and finally to binary files readable by Matlab . Each parameter consists of a two-column matrix with time in the first column and values in the second. Matlab 's ordinary plot functions have been used to build standard templates to speed up the first evaluation, and identify the time windows interesting for further analysis. The system identification has been per- 
formed via the System Identification Toolbox for Matlab (Ref. (3 )) that has shown to be a valuable general-purpose tool with a very user-friendly graphical user interface, and a good solution to avoid the time consuming development of a more application-tailored software package. The signals were low-pass filtered at $10 \mathrm{~Hz}$, and 5-10 seconds time windows selected for the identification. General input-output parametric models of the output-error type have been used, corresponding to the linear difference equation:

$$
y(t)=[B(q) / F(q)] u(t-n k)+e(t)
$$

being $\mathrm{y}(\mathrm{t})$ the output, $\mathrm{u}(\mathrm{t})$ the input and $\mathrm{e}(\mathrm{t})$ the noise; the number of delays nk had been previously identified with a dedicated test: the latency amounted initially to $400 \mathrm{~ms}$ due to a glitch in the communication protocol between the $\mathrm{CC}$ and the YACS interface, and has dropped now to $120 \mathrm{~ms}$ in the current architecture: this had no influence on the data quality, since it just produced a time shift of the output of known magnitude. The criteria to accept/refuse a model have been based on the minimization of the model order and on the analysis of the cross correlation function (between residuals and input), besides obviously on the best overall fit (minimization of the loss function). Once a satisfying model was found, it was exported to Matlab 's workspace and converted to continuous time. The remaining parameters have been identified uniquely according to a best-fit criterion. For the lateral and longitudinal translational dynamics the following model has been imposed:

$$
\begin{aligned}
& \dot{u}=X_{u} u-g \vartheta \\
& \dot{v}=Y_{v} v+g \varphi
\end{aligned}
$$

where the rotor flapping angles have been neglected, since not observable from the closed-loop behaviour. The Euler angles have been obtained by the integration of the angular rates $\mathrm{p}, \mathrm{q}$ and $\mathrm{r}$. The trim roll angle in hovering has been fixed to 4.5 degrees.

\section{RESULTS}

The response to step inputs of different amplitude have been processed, and the steady state gains identified to form the non-linear gains shaping the input. After that, linear transfer functions of general validity could be identified, as follows (degrees and gs as units):

$$
\begin{aligned}
\frac{\Delta p}{\Delta A I L} & =\frac{2.3 s\left(s^{2}+3.87 s+53.3\right)}{\left(s^{2}+6.29 s+16.2\right)\left(s^{2}+8.97 s+168\right)} \\
\frac{\Delta q}{\Delta E L E} & =\frac{0.5 s\left(s^{2}+9.76 s+75.5\right)}{\left(s^{2}+3 s+5.55\right)\left(s^{2}+2.06 s+123.5\right)}
\end{aligned}
$$

$$
\begin{aligned}
\frac{\Delta r}{\Delta R U D} & =\frac{9.7(s+12.25)}{9.7(s+4.17)\left(s^{2}+3.5 s+213.4\right)} \\
\frac{\Delta n_{Z}}{\Delta T H R} & =\frac{0.0828 s(s+3.37)}{(s+0.95)\left(s^{2}+13.1 s+214.1\right)}
\end{aligned}
$$

The attitude dynamics is well captured by the identified model. The YACS stabilizes the unstable phugoid mode, and produces pitch and roll behaviours that can be roughly approximated by first order responses characterized by a 0.7 seconds time constant (see Fig.3). A residual of the coupled fuselage/flapping/stabilizer-bar modes of the base platform can be found in the lightly damped modes at $11 \mathrm{rad} / \mathrm{s}$ (pitch) and $13 \mathrm{rad} / \mathrm{s}$ (roll). No physical interpretation is possible of the yaw dynamics, since it is heavily reshaped by the tail gyro. It can be observed anyway that a resonance mode around $2 \mathrm{~Hz}$ appears both in the yaw and in the vertical acceleration transfer function; the effect of this has been observed during the flight tests of an early version of the upper control system, that in certain conditions (tail wind) resulted into a $2 \mathrm{~Hz}$ coupled yaw/vertical acceleration oscillatory mode.

\section{LIMITATIONS}

The on-axis attitude dynamics is well captured by the model. The off-axis response has no practical importance for the closed-loop attitude dynamics, whereas an input in yaw produces as secondary effect a lateral acceleration that is not negligible; this is simply the effect of the free force generated by the tail rotor, that has not been modelled so far, since it becomes relevant only when the yaw input dominates the manoeuvre (for example during fast pirouettes). Having neglected the rotor flapping angles introduces an error in the steady state velocities. This has been observed in the results, but has been accepted since the error can be easily compensated with an integration loop in the speed controller; this loop would be anyway necessary to compensate the effect of wind. The model has been so far validated up to a forward speed of $50 \mathrm{~km} / \mathrm{h}$, and the simulated outputs are in good agreement with the flight data. However the velocity stability derivatives values will be refined through dedicated tests with a chase car, to increase the time available for stabilized flight. Despite not a limitation of the model, it has to be mentioned that the YACS is sensitive to continued accelerated flight, since its reference attitude angles (calculated) drift when accelerations are held for several seconds. 


\section{SIMULATION MODELS}

The above-described mathematical model has been implemented into:

- a set of Simulink models: SISO models are used for the first development of the control laws, while a complete 6 DOF for the verification process;

- a complete 6 DOF model written in $\mathrm{C}$ language for the verification of the complete control system; the model has been also integrated with a wind model and an auto-trim function for the initialisation of the simulations;

- a real-time C-language program running on the actual $\mathrm{CC}$, for the very last verification before flight; this latter implementation is a soft-implementation of the Hardware-In-theLoop concept: the complete flying hardware is involved by the simulations, but the servos' positions is not fed back into the mathematical model, that captures the closed loop behaviour and not the isolated platform dynamics.

These three simulations steps have shown to be somehow complementary, since stage a. has the advantage of flexibility and Simulink is a good development environment, stage b. allows debugging of the hand-written $\mathrm{C}$ code in faster-thenreal time, while stage $\mathrm{c}$. allows the final go/no-go decision for flight with a new software version. The possibility of visually checking the swash plate/tail servo behaviour during simulated flight on ground has shown to be a very powerful feature.

\section{CONCLUSIONS}

A mathematical model of the stabilized Yamaha RMAX remotely piloted helicopter, based on flight test results, has been developed. The builtin stabilization system (YACS) effectively stabilizes the attitude dynamics and practically decouples the responses to the pilot's sticks, which control the pitch angle, the roll angle, the yaw rate and a pseudo vertical velocity. Despite not suitable for extremely aggressive manoeuvring, the YACS can be successfully used as inner loop by an autonomous navigation system, as also demonstrated by the ongoing test activity carried out by the UAVTech research group $((1))$.

\section{FUTURE WORK}

The mathematical model above described has already fulfilled the needs of the project, since outer control loops (up to full autonomy) have already been developed and successfully flighttested (Ref. $(2)$ ). The model allowed a very efficient design process of the control system, since empirical tuning has almost never been necessary. As only improvement, the speed derivatives will be refined, through dedicated tests. The focus of the simulation group will now shift to the identification of the dynamics of the base platform (YACS disengaged), to support the design of a new attitude control system, more suitable to highly manoeuvred flight and exportable to other platforms.

\section{REFERENCES}

[1] G. Conte, S. Duranti, and T. Merz. Dynamic 3D path following for an autonomous helicopter. In Proc. of the 5th IFAC Symposium on Intelligent Autonomous Vehicles, July 2004.

[2] P. Doherty. Advanced research with autonomous unmanned aerial vehicles. In Proc. of the 9th International Conference on the Principles of Knowledge Representation and Reasoning, pages 731-732, June 2004.

[3] Lennart Ljung. System Identification Toolbox User's Guide For Use with Matlab. The MathWorks, 1997.

[4] M. Tischler, B. Mettler, and T. Kanade. System identification modeling of a model-scale helicopter. CMU-RI-TR-00-03, 2000.

[5] Yamaha Motor Co., Unmanned Helicopters. http://www.yamahamotor.co.jp/global/industrial/sky/index.html. 


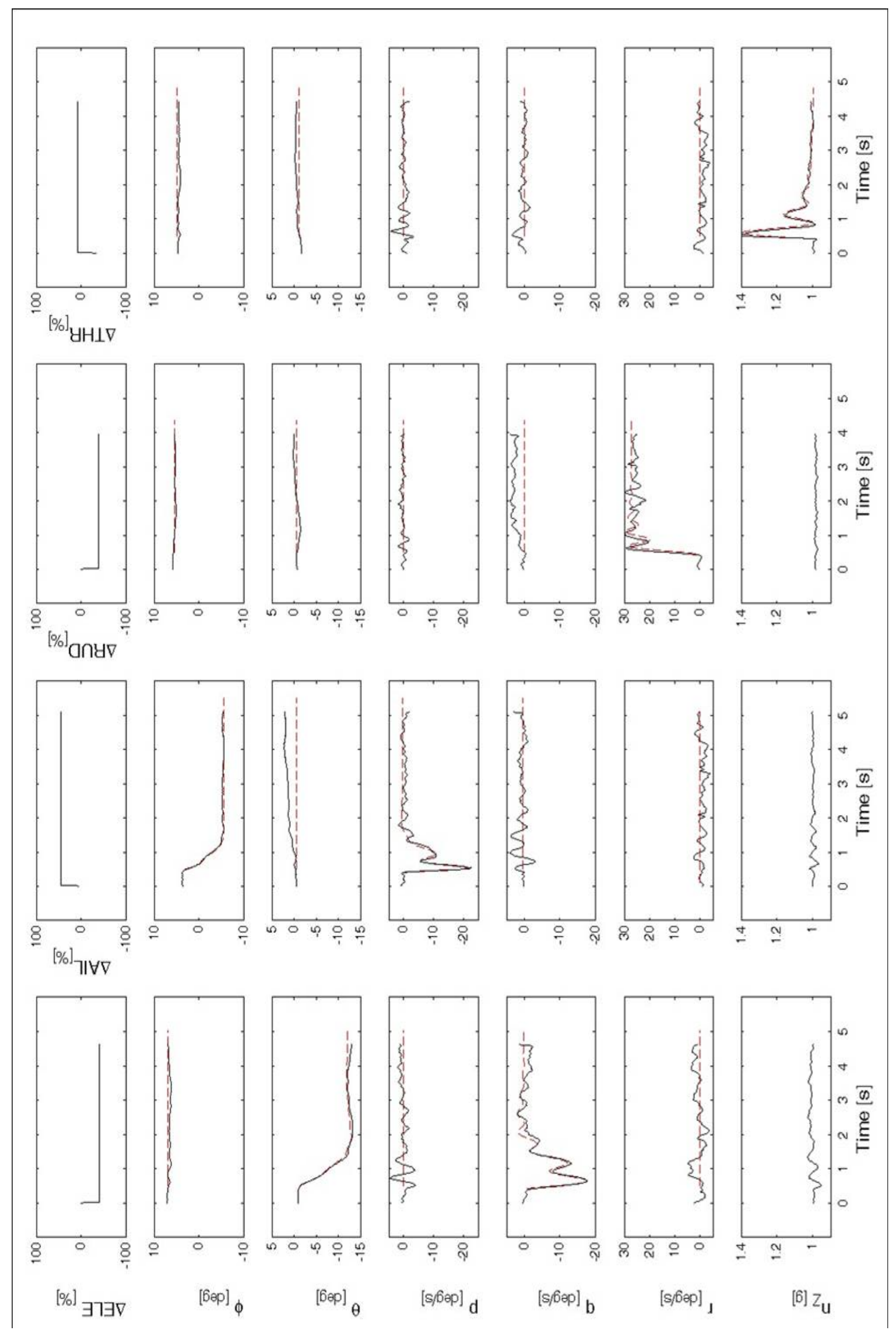

Fig. 3. Time domain model verification (model $=$ dashed line, flight test data $=$ continuous line) 